\title{
On the Mechanism of Factory-Roof Formation
}

\author{
J. Pokluda, K. Slámečka and P. Šandera \\ Faculty of Mechanical Engineering, Brno University of Technology, Technická 2, \\ 61669 Brno, Czech Republic, pokluda@fme.vutbr.cz
}

\begin{abstract}
The investigated factory-roof (F-R) patterns were produced by cyclic torsion loading of $V$-notched cylindrical specimens made of the high-strength low-alloy steel. A three-dimensional model of the F-R pattern was constructed by means of the stereophotogrammetry. This allowed us to recognize the geometrical proportions as well as to understand the basic geometrical rules of its formation. The theoretical analysis revealed that the F-R initiates by elementary mode I branches at the front of semi-elliptical surface cracks. The exact positions of such branches can be analytically determined in terms of the maximum synergy of mode II and mode III loadings. An increasing density of semi-elliptical cracks initiated on the surface results in the refinement and the size reduction of $F-R$ patterns. This effect can, together with strong wear damage, explain the fact that the F-R patterns are usually not observed in the lowcycle fatigue region.
\end{abstract}

\section{INTRODUCTION}

The factory-roof (F-R) indisputably belongs to the most extraordinary fractographical patterns in fatigue and fracture of metallic materials. Up to now, this nearly periodical and very complicated fracture morphology was observed only in notched cylindrical specimens subjected to fatigue torsion loading. The roughness (or visibility) of F-R particularly depends on the applied cyclic shear stress amplitude though a significant influence of both the material microstructure and the material yield strength was also observed (e.g. [1-6]). In spite of the fact that first reports on the F-R already appeared in early 1950's, both the detailed formation mechanism and the related quantitative rules are still unknown. Consequently, many principal questions concerning the F-R phenomenon remain unsolved. The recently published experimental work [7] suggested that there are three stages of F-R formation: (i) initiation and growth of surface semielliptical microcracks under shear loading modes II+III, (ii) their interaction, coalescence and growth in the local mixed mode I+II+III by forming mode I branches (tilted and twisted segments) (iii) growth of the periodical main crack under the prevalent mode I loading. The theoretical works $[8,9]$ were focused on the problem of friction and shielding phenomena associated with a simple saw-tooth model of F-R patterns. These findings were, however, not completed by any quantitative threshold conditions that could explain the position of initiation steps and the growth kinetics of F-R. To our knowledge, there is also a lack of any detailed 3D topographical analysis 
of these patterns. Therefore, the mechanism of F-R seems to be still very challenging for a further research.

This paper attempts to fill the gap of our knowledge about the process of F-R formation and tries to answer some of the basic questions such as:

(i) What is the characteristic 3D picture of factory roofs?

(ii) Which physically based relationships control the initiation and growth of F-R?

(iii) What is the kinetics of F-R formation?

(iv) Why the factory roofs are not observed in the region of a very low cycle fatigue?

In order to answer these questions, a 3D model of a characteristic F-R morphology must be constructed. Moreover, a geometrically consistent and physically justified model of the F-R formation is to be developed.

\section{THEORETICAL BASIS AND EXPERIMENTAL METHODS}

\section{Remarks on propagation of mixed-mode cracks}

The crack initiation process usually starts on the surface by formation of nearly planar semi-elliptical cracks propagating under the mixed-mode II+III dominance. The mode II+III cracks keep the in-plane growing for a certain time in spite of the relation $K_{I I}=0.87 K_{I}$, where $K_{I I}$ is the stress intensity factor for the mode II crack and $K_{I}$ is that for its $70.5^{\circ}$ mode I branch [10]. This is caused by several reasons. As it is well known from the push-pull regime, the threshold stress intensity factor for propagation of small (short) shear cracks is significantly lower than that for the long mode I cracks. There is also a high shear stress concentration in front of the sharp notch that is constraint to the maximum shear plain. Nevertheless, the nearly straight crack front of semi-elliptical cracks changes to a highly tortuous profile by inclinations towards a much higher mode I loading rather soon. This dramatic change is accomplished by tilting (branching) or twisting of the crack front segments.

There are several possible reasons for such behaviour that are particularly related to the crack advance in the radial direction. Indeed, the notch stress concentration decreases and, moreover, there is also a very limited ability of a pure mode III segment to propagate in that direction [11]. Therefore, the aspect ratio of the semi-elliptical cracks becomes rather high. Interactions of the crack front with microstructural barriers (grain or phase boundaries) are accompanied by an increase in the roughness of crack flanks. This leads to an increase in the friction stress (shear closure) and to the reduction of the mode II+III crack driving force. In order to get a maximum support of the opening mode I to avoid the above mentioned problems, the mode II segments rotate around the axis parallel to the crack front. Such a rotation is relatively easy even for large tilted segments since their planes intersect the main crack plane along the line (curve). On the other hand, twisting around the axis perpendicular to the crack front provides the mode III crack segments with the mode I support. Since the planes of the twisted elements and the main crack intersect only in one point, the size of the twisted elements is very limited and the twisting can occur only on microscopic ledges at the main crack front. This means that the formation of mode I branches at the mode II 
cracks is easier than that at the mode III cracks. In any case, the F-R formation starts by the creation of mode I branches at particular sites along the elliptical mixed-mode II+III front (see hereafter).

The following relationships describe the stress intensity factors for the mode I branch under pure mode II and mode III loading:

$$
\begin{gathered}
k_{1, I I}=\frac{3}{2} K_{I I} \cos \frac{\beta}{2}(3 \cos \beta-1), \\
k_{1, I I I}=K_{I I I} \sin 2 \gamma,
\end{gathered}
$$

where $\gamma$ is the rotation angle around the perpendicular axis and $\beta$ is the rotation angle around the parallel axis [10]. Factors $K_{I I}$ and $K_{I I I}$ along the semi-elliptical crack front can be determined as

$$
\begin{aligned}
& K_{I I}=\frac{\tau \sqrt{\frac{\pi b^{3}}{a}} k^{2} \cos \alpha}{\left(\left(k^{2}-v\right) e l E+v k^{\prime 2} e l K\right)\left(b^{2} \cos ^{2} \alpha+a^{2} \sin ^{2} \alpha\right)^{1 / 4}}, \\
& K_{I I I}=\frac{\tau \sqrt{\pi a b} k^{2} \sin \alpha}{\left(\left(k^{2}-v\right) e l E+v k^{\prime 2} e l K\right)\left(b^{2} \cos ^{2} \alpha+a^{2} \sin ^{2} \alpha\right)^{1 / 4}},
\end{aligned}
$$

where $\alpha$ is the angle defined by the related circle (see Fig. 4 hereafter), $a$ and $b$ are the half-axes of the semi-ellipse, $k=\sqrt{1-\frac{b^{2}}{a^{2}}}, k^{\prime}=\frac{b}{a}$, elK is the complete elliptic integral of the first kind of the variable $k$, elE is the elliptic integral of the second kind [12]. However, the mixed-mode II+III exists in all points of the semi-elliptical crack front except for two points on the surface (pure mode II) and one point at its centre (pure mode III). Thus, the stress intensity factors for possible branched elements depend on angles $\alpha, \beta, \gamma$ and, in particular, on the angles $\chi$ and $\vartheta$ that respectively define the twist and the tilt of the branch with respect to the semi-elliptical crack front. Consequently, the solution of the branch problem is not trivial and the branch of a maximal effective stress intensity factor $K_{\text {eff }}$ must be found by a rather complicated mathematical procedure. Hereafter, the angles corresponding to such a branch are denoted $X$ and $\Theta$.

\section{Experimental methods for determination of $3 D$ fracture topography}

Two methods based on different physical principles were used to reconstitute the threedimensional micromorphology of F-R.

The stereophotogrammetry is a method that makes use of the stereoscopic principles in order to obtain topographical data of fracture surface under investigation. Inputs of the method are two images of the analyzed region taken from different positions of view 
(so-called stereoimages, stereopair) and some additional parameters that characterize a projection used during their acquisition. Usually, a scanning electron microscope (SEM) equipped with the eucentric holder is employed and the stereopair is obtained by tilting the specimen in the microscope chamber by an angle that depends on a local roughness of the surface. The stereopair is processed via a matching algorithm in order to find corresponding points on both images (homologue points) and the relative $z$-coordinates of homologue points are calculated. The 3D model of depicted surface area usually consists of 10-20 thousand non-equidistant points and so called Delaunay triangulation must be performed [12].

The optical chromatography represents another method useful for the 3D reconstruction of the fracture surface micromorphology. The profilometer MicroProf FRT, Fries Research \& Technology GmbH is based on utilization of the chromatic aberration of the optical sensor CHR $150 \mathrm{~N}$ positive lens. Different light monochromatic components are focused in different heights from a reference plane at the output of the optical fibre. The light intensity exhibits a maximum at the wavelength exactly focused on the surface and the height of the surface irregularities is deduced by using a calibration table. This optical method was used only for verification of selected results obtained by the stereophotogrammetry.

\section{EXPERIMENTAL RESULTS}

The fracture surfaces containing F-R patterns were generated by the reversed torsion loading of $\mathrm{V}$-notched cylindrical specimens (the diameter of $10 \mathrm{~mm}$, the notch depth of $1 \mathrm{~mm}$, the notch radius of $0.3 \mathrm{~mm}$, the notch angle of $45^{\circ}$ ) made of a high-strength lowalloy steel by means of the mechanical stand MZGS 200. The steel was heat treated to obtain a fine bainitic microstructure. Basic mechanical properties of the material are shown in Table 1. Three values of the torsion moment $M_{t 1}=13 \mathrm{Nm}, M_{t 2}=17.9 \mathrm{Nm}$ and $M_{t 3}=22.7 \mathrm{Nm}$ led to the fatigue lifes of $N_{f 1}=8.31 \times 10^{5}, N_{f 2}=2.44 \times 10^{5}$ and $N_{f 3}=1.58 \times 10^{4}$ cycles, respectively. The macrophotographs of the related fracture surfaces are shown in Fig. 1. Unlike in the low-cycle fatigue case, distinct F-R patterns were observed in both high-cycle regimes.

Table 1. Mechanical properties of the HSLA steel.

\begin{tabular}{ccc}
\hline $\begin{array}{c}\text { Yield stress } R_{e} \\
(\mathrm{MPa})\end{array}$ & $\begin{array}{c}\text { Ultimate stress } R_{m} \\
(\mathrm{MPa})\end{array}$ & $\begin{array}{c}\text { Threshold } \Delta K_{t h}(R=-1) \\
\left(\mathrm{MPa} \cdot \mathrm{m}^{1 / 2}\right)\end{array}$ \\
\hline 840 & 950 & 9.2 \\
\hline
\end{tabular}

Fig. 1 documents not only the complexity but also a certain regularity of F-R patterns. Indeed, the lamellar-like F-R structure consists of nearly parallel elongated "mountain-like" massifs (segments). Rather narrow valleys with secondary cracks join the individual massifs. Near the surface, the massif is usually split into two smaller 
segments. These small segments, constituting the initial stage of the F-R formation, eventually join to create the main individual massifs. Note that there are several F-R patterns which significantly differ in their size. The smaller are the F-R patterns the finer is their lamellar structure.
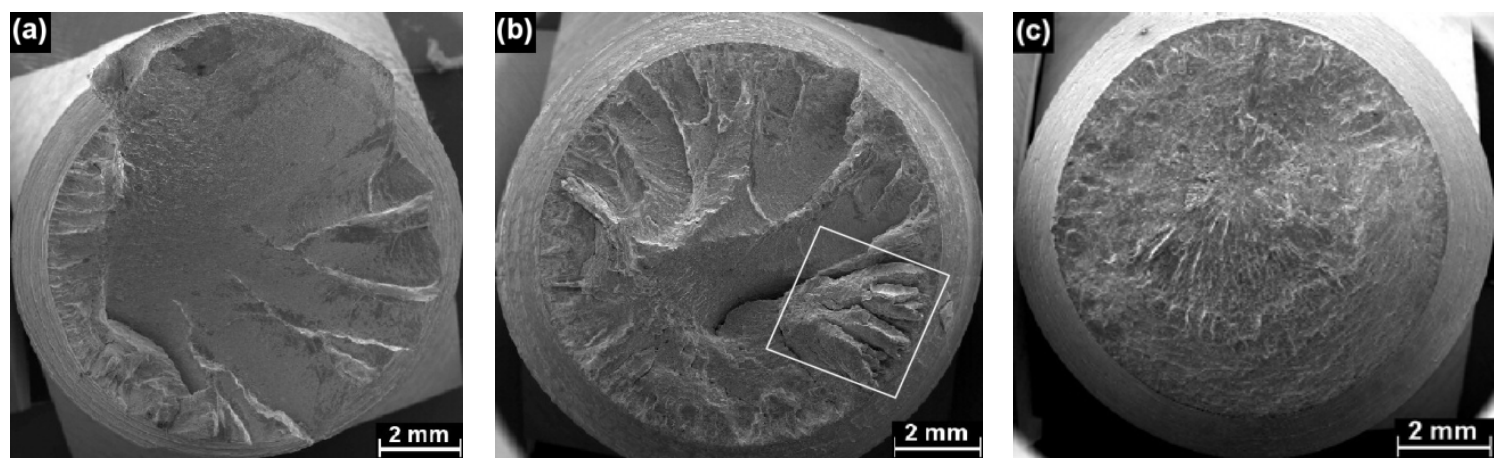

Figure 1. Fracture surfaces of specimens with the factory-roof patterns.

(a) $M_{t 1}=13 \mathrm{Nm}\left(N_{f 1}=8.31 \times 10^{5}\right.$ cycles $)$; (b) $M_{t 2}=17.9 \mathrm{Nm}\left(N_{f 2}=2.44 \times 10^{5}\right.$ cycles $)$;

(c) $M_{t 3}=22.7 \mathrm{Nm}\left(N_{f 3}=1.58 \times 10^{4}\right.$ cycles $)$.

The investigated F-R region is highlighted on the middle picture.

In Fig. 2, the network of profile projection lines on the segments corresponding to the F-R patterns highlighted in Fig. $1 \mathrm{~b}\left(M_{t 2}=17.9 \mathrm{Nm}\right)$ is depicted. The corresponding profiles in nearly tangential and radial directions are plotted in Figs. 3a, b and c. The profiles well document the general geometrical features: The slopes (hillsides) of the embryonic triangular-like segments gradually decrease starting from an initial angle of $65^{\circ}-77^{\circ}$ near the surface (e.g. the profile 3 ) to somewhat lower angles of $60^{\circ}-71^{\circ}$ near their conjunction to one main segment. This stepwise decrease of the slope continues to about $30^{\circ}-50^{\circ}$ at the top of the main ridge (near the profile 6) and finally to about $30^{\circ}$ at the segment near the centre of the fracture surface.

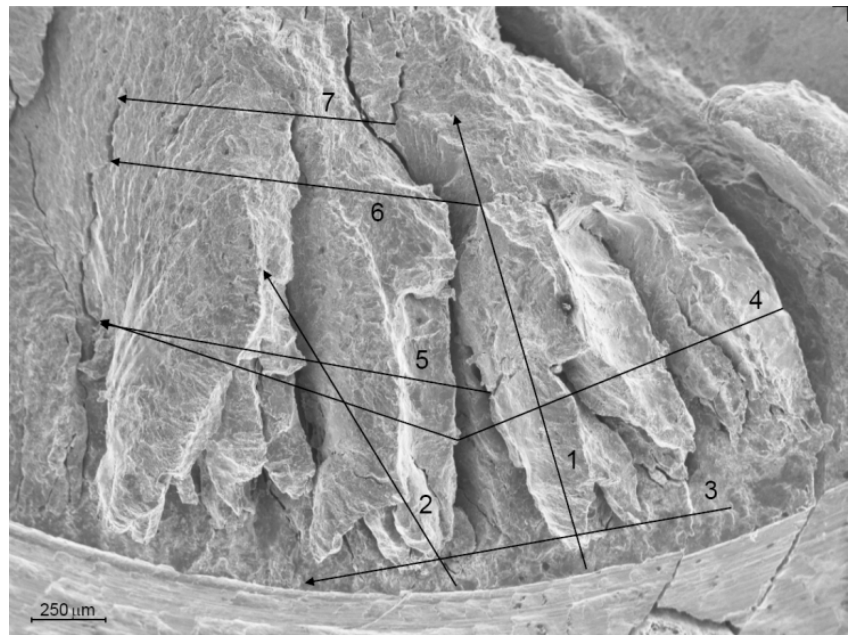

Figure 2. The projection lines of profiles utilised for the stereophotogrammetrical analysis. 
(a)

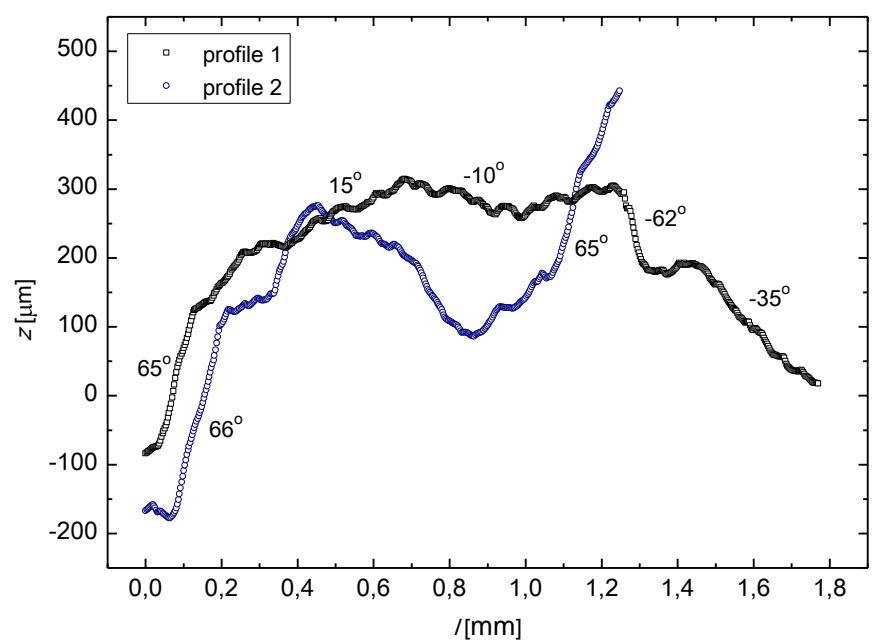

(b)

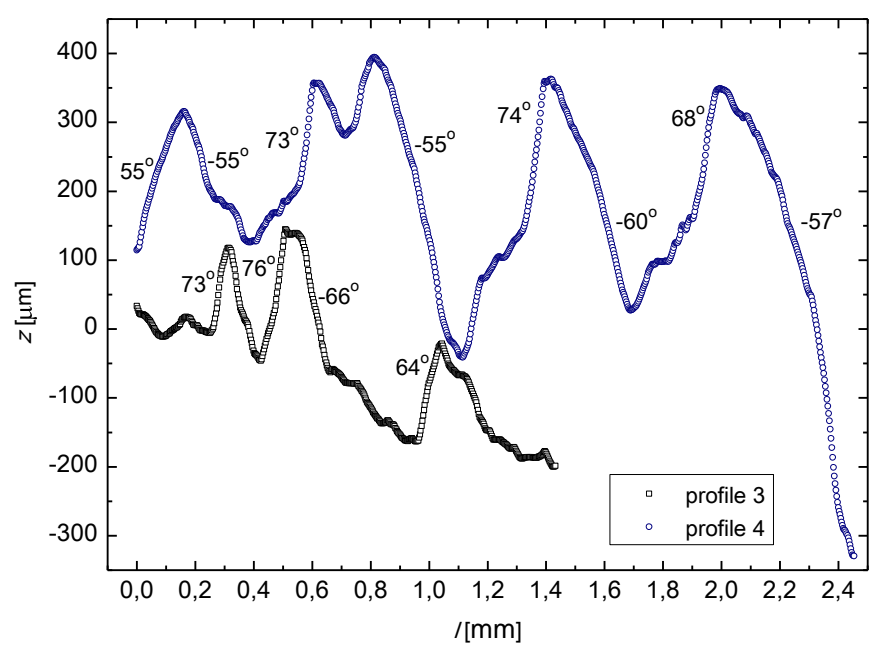

(c)

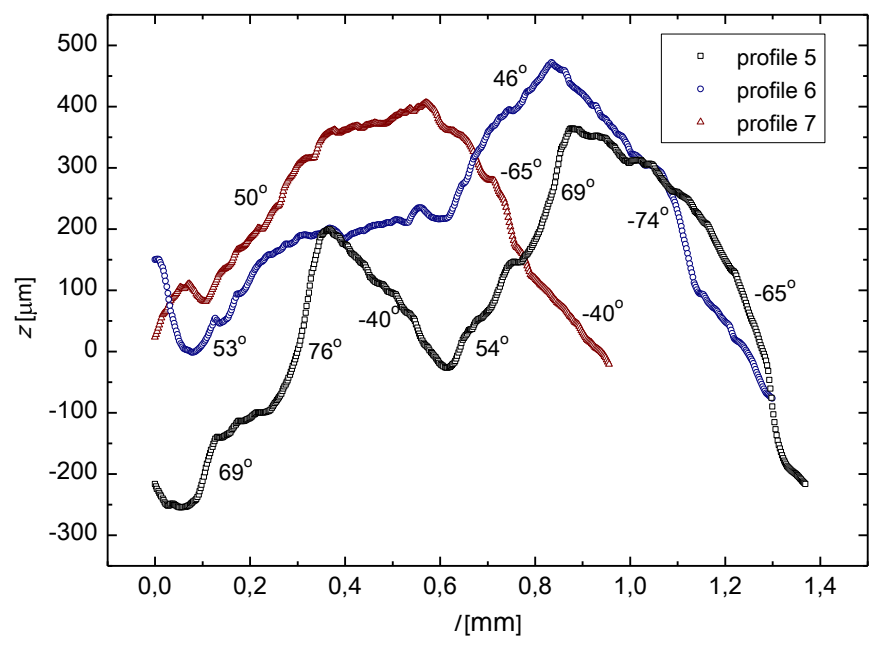

Figure 3. Topographical profiles corresponding to the projection lines in Fig. 2. 
The profile 3 clearly demonstrates that, in fact, the planes of semi-elliptical cracks in between the F-R nuclei are microscopically tortuous. Both the width and the height of the nuclei initially increase up to the conjunction site. After the coalescence, the dimensions of the main segment slowly decrease approximately following the geometry of converging radials as defined by the F-R segments (the circle sectors). Thus, both the height and width of the F-R patterns can be considered to be closely associated with their distance by a rather simple geometrical rule. In particular, the global size of the F$\mathrm{R}$ pattern decreases with increasing density of individual segments (see Fig. 1).

The fracture micromorphology was damaged by rubbing wear due to a repeated contact of fracture surfaces and the related bending loading. This loading initiates secondary cracks and contributes also to a further mode I propagation of the main F-R crack inside the specimen. Therefore, numerous wear traces (fibre patterns or tire tracks) could be found on the SEM pictures of the fracture surface. Nevertheless, few striation fields identifying the local propagation of mode I crack front were also discovered in advanced growth stages.

\section{THEORETICAL RESULTS}

The main aim of the theoretical analysis was to predict the most probable sites of the mode I branching at the semi-elliptical crack front. This analysis utilized eqs. (1) - (4) in order to find the angles $X$ and $\Theta$ associated with a branch of a maximal $K_{\text {eff }}$ (or $K_{I}$ ) as functions of the polar angle $\varphi$ that defines the position at the semi-elliptical crack front - see Fig. 4. The branch plane and the planes containing directions of pure applied mode II and mode III loadings (normal and tangential to the semi-ellipses) are generally different. The angles $\beta$ and $\gamma$ which define the mutual inclinations of those planes with respect to projected mode II and mode III directions were utilized and the relationships $\tan \beta=\tan \vartheta \cos \chi \quad$ and $\tan \gamma=\tan \vartheta \sin \chi \quad$ describing the necessary geometrical transformation were found.

A detailed description of the whole mathematical procedure would significantly exceed the permitted extent of the article and, therefore, only final results can be reported here. For the branch $(b / a=0.7)$ of a maximal effective driving force the analysis revealed that the tilt angel $\Theta \approx 71.6^{\circ}$ keeps the same value within the whole range of $\varphi \in\left\langle 0,180^{\circ}\right\rangle$ and the twist angle $X \approx 19.5^{\circ}$ remains to be constant within the range $\varphi \in\left\langle 0^{\circ}, 54^{\circ}\right\rangle$ or $\varphi \in\left\langle 126^{\circ}, 180^{\circ}\right\rangle$. Simultaneously, such a branch is loaded by a maximal pure local mode I. Starting from the critical polar angle $\varphi_{c}=54^{\circ}$, the twist angle $X$ decreases to reach a zero value for $\varphi=90^{\circ}$. This result holds well for all semi-elliptical cracks exhibiting the aspect ratio in the range $b / a \in\langle 0.6,0.8\rangle$ that contains a great majority of experimentally observed semi-elliptical cracks. Only the critical polar angle changes in the range $\varphi_{c} \in\left\langle 45^{\circ}, 60^{\circ}\right\rangle$. The scheme elucidating the position and orientation of a 
selected elementary branch is shown in Fig. 4 along with the link between the angles $\varphi$ and $\alpha$.

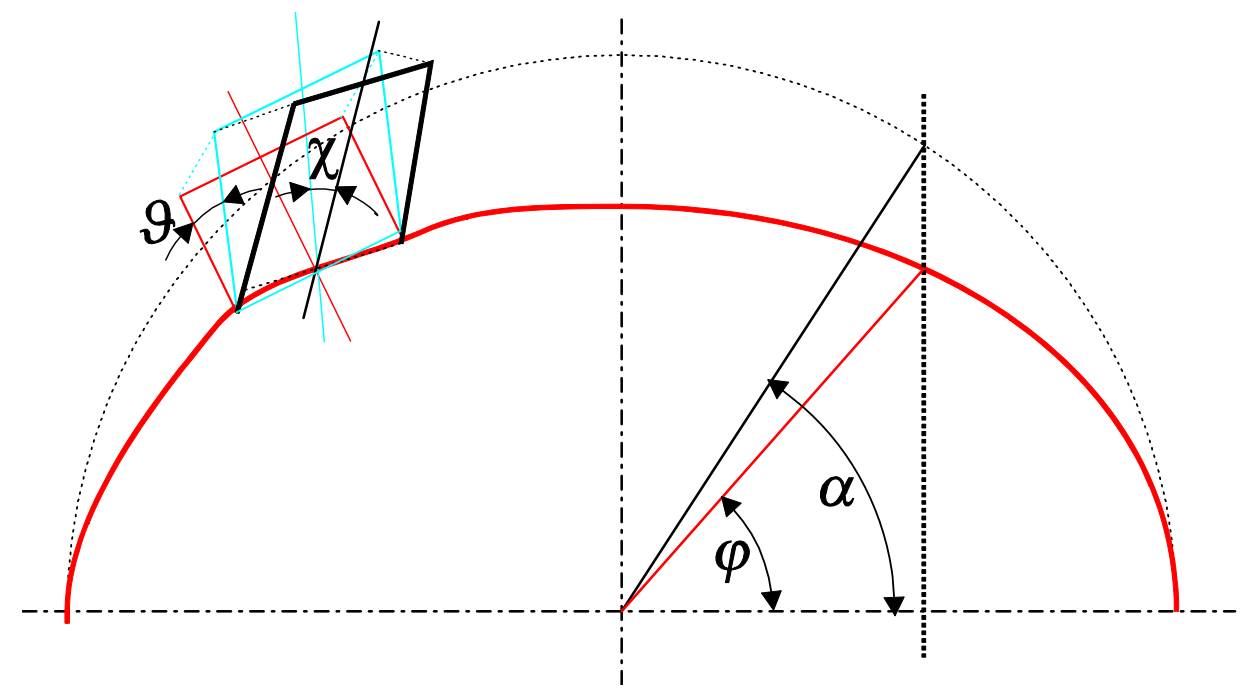

Figure 4. Scheme of a branched element at the semi-elliptical crack front.

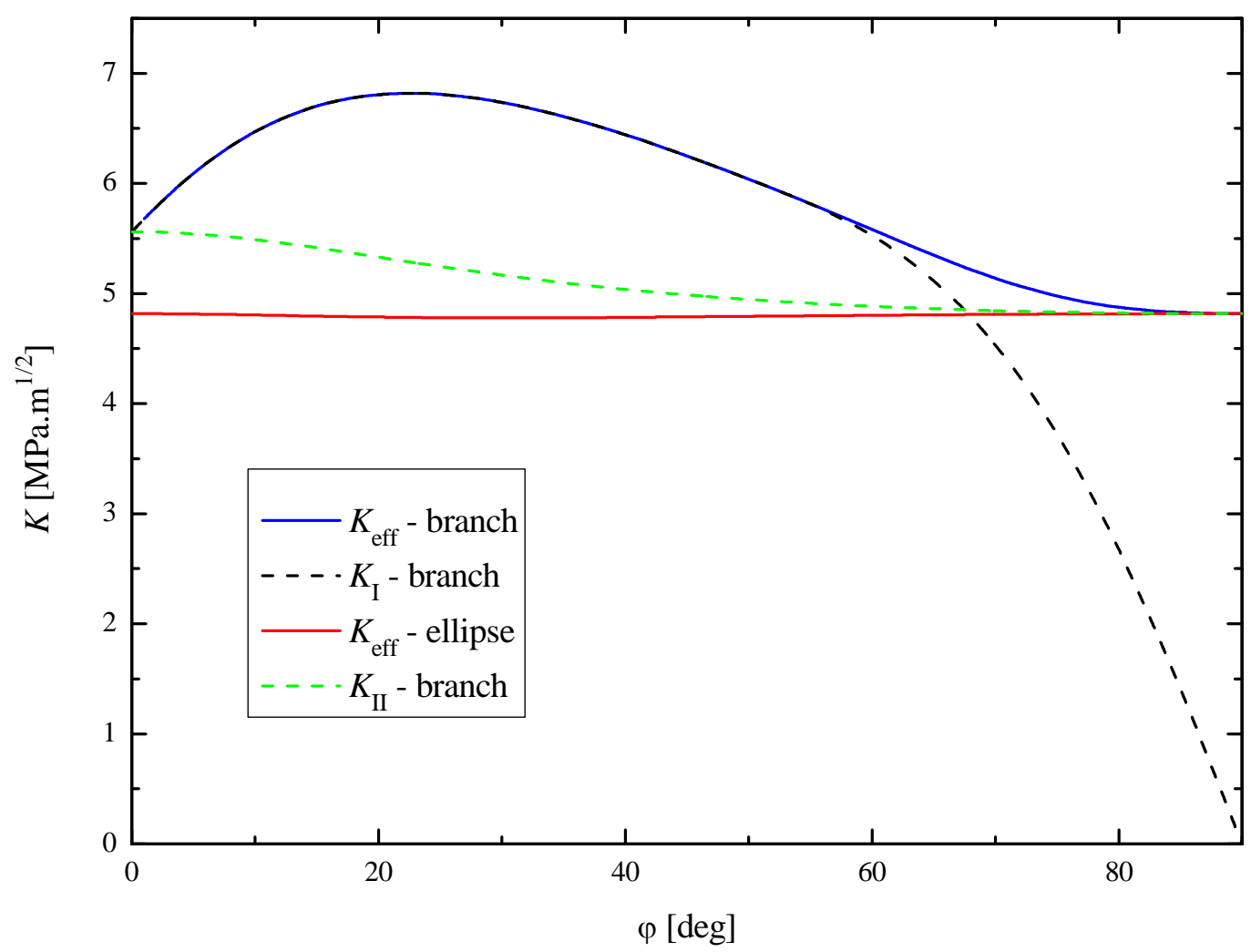

Figure 5. The solution of the branched crack problem for the semi-elliptical crack front with $b / a=0.7$. 
The dependences of various modes of the stress intensity factor on the angle $\varphi$ are plotted in Fig. 5 for the semi-elliptical crack with an aspect ratio $b / a=0.7$. The value $M_{t 2}=17.9 \mathrm{Nm}$, the relevant notch stress concentration factor $k_{v}=1.94$ [13] and the averaged value $a=150 \mu \mathrm{m}$ were used in correspondence with the investigated case (see Fig. 6). The most important finding is that the superposition of tilting and twisting raises the values of the effective stress intensity factor $K_{\text {eff }}$ for the mode I branch (the blue curve) significantly above the original values for the semi-elliptical crack front (the red curve). The contribution of the mode II to the $K_{\text {eff }}$-factor for the mode I branch is shown by the green curve whereas that of the mode III is given by the difference between the blue and the green curve. These results can be understood in terms of a synergy effect of both the mode II and the mode III loading on the creation of the mode I branch. Within the range $\varphi \in\left\langle 0,54^{\circ}\right\rangle$ the values of $K_{\text {eff }}$ and $K_{I}$ (black line) are identical and, for all the semi-elliptical cracks, their maxima lie in the corresponding range $\varphi_{\max } \in\left\langle 20^{\circ}, 30^{\circ}\right\rangle$. This range determines two segments on the semi-elliptical crack front that correspond to the maximal probability of the creation of the F-R nuclei (the mode I branches). In Fig. 6, the ranges of $\varphi_{\max }$ are marked on the crack fronts of all deduced semi-elliptical cracks. One can see that there is a good correspondence with practically all the real initiation sites of F-R nuclei.

The creation and further propagation of F-R nuclei is conditioned by exceeding the mode I threshold for the HSLA steel on the mode I branches. For cracks with $b / a \in\langle 0.6,0.8\rangle$, the maximal values of $K_{I}$ are in the range $K_{\max } \in\langle 6.4,7.1\rangle \mathrm{MPa} \cdot \mathrm{m}^{1 / 2}$. These values are higher than the threshold amplitude $\Delta K_{t h} / 2=4.6 \mathrm{MPa} \cdot \mathrm{m}^{1 / 2}$ but still sufficiently close to the threshold. This result, along with the good prediction of initiation sites, confirms the plausibility of the theoretical approach.

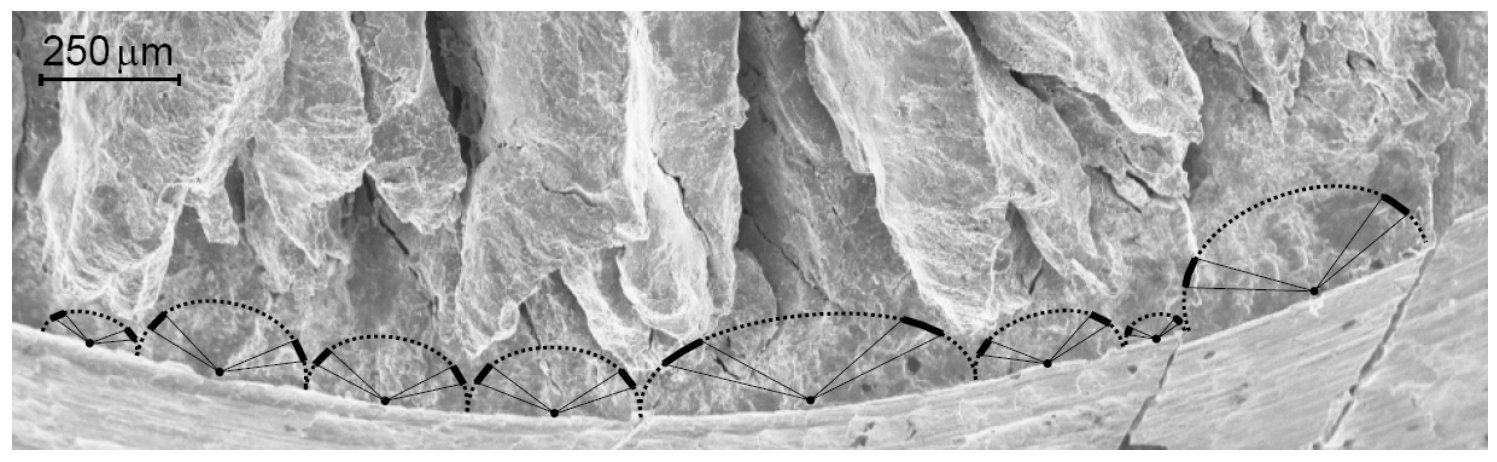

Figure 6. Illustration of the correspondence between the predicted positions of the mode I branch at semi-elliptical crack fronts and real position of the F-R nuclei.

\section{MODEL OF FACTORY-ROOF FORMATION}

On the basis of above mentioned experimental and theoretical results, the kinetics of F-R formation can be qualitatively assessed. Individual stages of F-R formation in terms 
of gradual positions of F-R crack front are shown in Fig. 7, where a 3D image of the investigated F-R is depicted. The process starts with the creation of mode I branches at defined sites of semi-circular cracks. In the cases of considerable size difference of neighbouring semi-elliptical cracks the mode I branch appears first on the larger semiellipse. When a large semi-ellipse is adjacent to a small one, a short stage of a backward growth towards both the critical site at the smaller semi-ellipse and the remaining semielliptical crack front may appear due to their mutual interaction. This accelerates both the initiation of the second mode I branch and the coalescence process.

After the local coalescence, the initial F-R crack front consists of the local spatial ledges and branches that form the embryonic massifs connected by the remaining fronts of semi-elliptical cracks. The contact bending loading on these massifs starts to produce the secondary cracks adjacent to the nuclei and, later on, to the main valleys (or hilltops on the mating fracture surface). These mode I cracks spread in planes inclined by $45^{\circ}$ to the macroscopic (horizontal) plane of the maximum shear stress, and eventually approach the advancing F-R crack front. In order to reduce the line tension of such a tortuous front, the embryonic massifs expand in both the radial direction (inside the specimen bulk) and the tangential direction (along the semi-elliptical fronts). In this way, both the width and the height of the nuclei increase while forming the local U-shaped valleys of a decreasing width. This finally leads to a coalescence of the embryonic segments (or a termination of the local valleys) to form the main massifs.

After this coalescence, the main front of a nearly saw-tooth profile propagates further in the direction of a maximum increase of the crack driving force, i.e. more or less in the radial direction. For a clear geometrical reason, the F-R crack front has to contract during the propagation towards the specimen centre. Consequently, the main massifs are brought mutually closer and their heights and widths decrease. The extinction of F-R patterns near the specimen centre eventually precedes the final fracture of the specimen.

When considering all the presented theoretical and experimental results, the first three questions raised in the introduction seem to be answered in a satisfactory manner. The last question can also be answered in a rather simple way. The density of initiated semi-elliptical cracks increases with decreasing number of cycles to failure (increasing applied stress range). For the above described geometrical reasons, the higher the density, the lower the size (and height) of the F-R patterns. This means that the F-R practically vanish when approaching the region of a very low cycle fatigue. In this region, moreover, the shear displacements become very large and the related strong wear causes a total destruction of the fine F-R morphology.

Let us finally remark that the torsion which induces the biaxial stress state is not the only one kind of loading that produces the F-R patterns. Indeed, these patterns are developing also under a pure shear loading, i.e. under a uniaxial stress state [14]. 


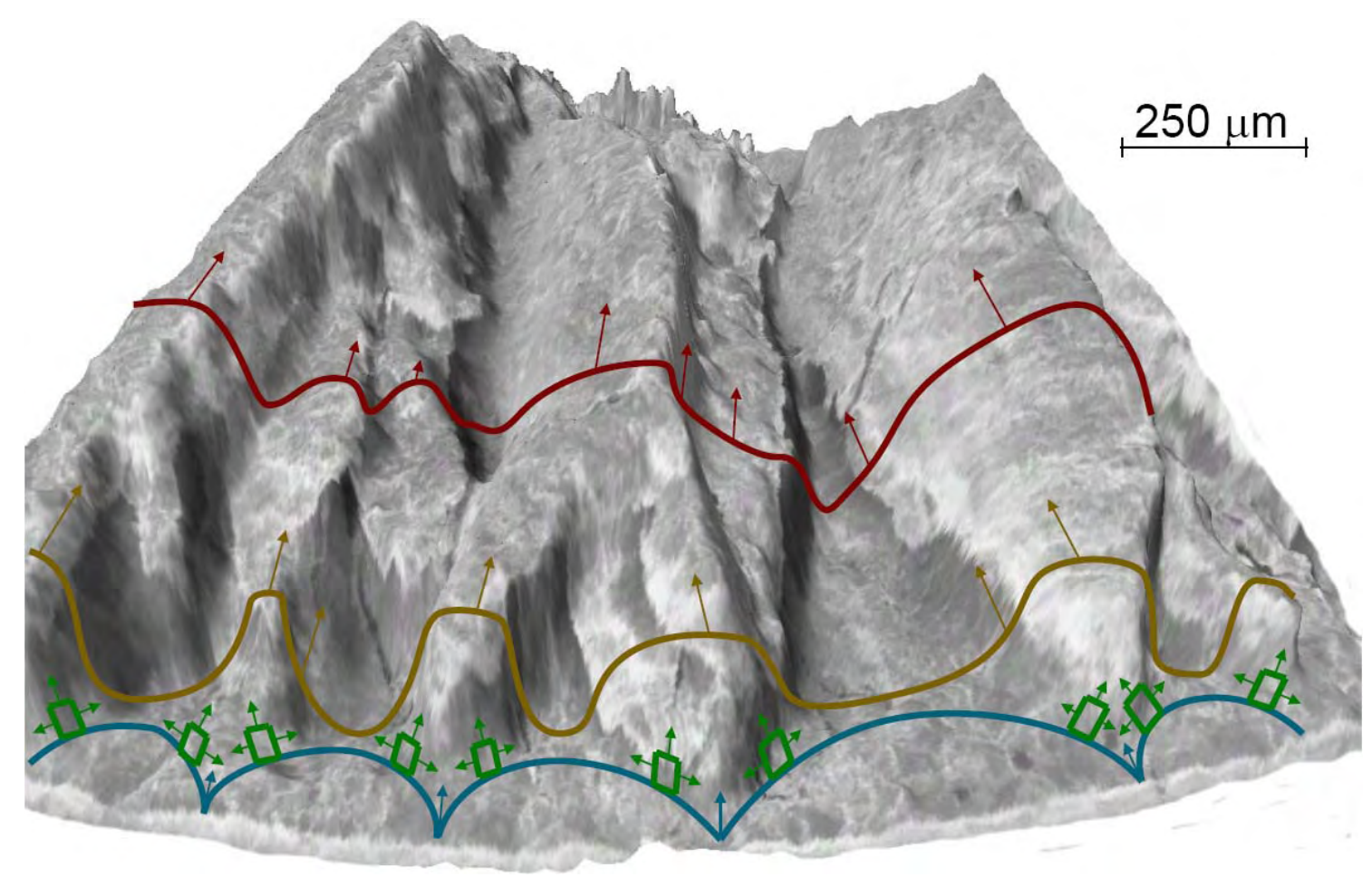

Figure 7. The 3D model of the F-R formation. Because of the perspective view, the scale-bar corresponds only to the region of semi-elliptical cracks.

\section{CONCLUSIONS}

The main results of the experimental and theoretical investigation of the factory-roof formation can be summarized in the following points:

(i) A three-dimensional picture of the F-R pattern was constructed by using the stereophotogrammetry. This allowed us to recognize the geometrical proportions as well as to understand the basic geometrical rules of its formation.

(ii) The F-R initiates by a creation of elementary mode I branches at particular points along the fronts of semi-elliptical surface cracks. Positions of these points can be analytically determined in terms of the maximum synergy effect of the mode II and the mode III loading.

(iii) An increasing density of initiated semi-elliptical cracks results in the refinement and the size reduction of F-R patterns. This phenomenon, together with the wear caused by high shear displacements, constitutes the main reason why the F-R patterns cannot be observed in the region of a very low cycle fatigue.

\section{Acknowledgment}

This work was supported by the Ministry of Youth, Education and Sports of the Czech Republic in the frame of the project MSM0021630518 and by Czech Science Foundation (project 106/08/P366). Authors thank also to Prof. R. Pippan who has 
kindly enabled extended stereophotogrammetrical measurements at the Erich-Schmid Institute of Materials Science in Leoben, Austria.

\section{REFERENCES}

1. Nayeb-Hashemi, H., McClintock, F.A. (1982) Metall. Trans. A 13 2197-2204.

2. Ritchie, R.O., McClintock, F.A., Nayeb-Hashemi, H. and Rittler M.A. (1982) Metall. Trans. A 13 101-110.

3. Tschegg, E.K. (1982) Mater. Sci. Eng. A 54 127-137.

4. $\quad$ Nayeb-Hashemi, H., McClintock, F.A. and Ritchie, R.O. (1983) Int. J. Fract. 23 163-185.

5. Multiaxial Fatigue (1985) Miller, K.J., Brown, M.W. (Eds) ASTM STP 853, ASTM, Philadelphia.

6. Tanaka, K., Akinawa, Y. and Yu, H. (1999) In: Mixed-Mode Crack Behaviour Miller, K.J., McDowel, D.L. (Eds.), ASTM STP 1359, ASTM, West Conshohocken.

7. Makabe, C., Anggit, M., Sueyoshi, T. and Yafuso, T. (2006) J. Test. Eval. 34 423-429.

8. Vaziri, A., Nayeb-Hashemi, H. (2005) Engng. Fract. Mech. 72 617-629.

9. Vaziri, A., Nayeb-Hashemi, H. (2006) Int. J. Sol. Struct. 43 4063-4081.

10. Pook, L.P. (2002) Crack paths, Wit Press, Southampton.

11. Pokluda, J., Pippan, R. (2005) Fat. Fract. Engng. Mater. Struct. 28179 - 186.

12. Kasir M. K., Sih G. C. (1966) J. Appl. Mech. 33 601-611.

13. Pilkey, W.D., Pilkey, D.F. (2008) Peterson's Stress Concentration Factors, John Willey \& Sons, New York.

14. Holáň, L., Pippan, R., Pokluda, J., Horníková J., Hohenwarter, T. and Slámečka K. (2009) In: Crack Paths 2009, Vicenza (accepted). 